\title{
BMJ Open Effects of prismatic adaptation on balance and postural disorders in patients with chronic right stroke: protocol for a multicentre double-blind randomised sham-controlled trial
}

\author{
Aurélien Hugues (D) , ${ }^{1,2,3}$ Amandine Guinet-Lacoste, ${ }^{1,2,3}$ Sylvie Bin, ${ }^{4}$ \\ Laurent Villeneuve, ${ }^{4,5}$ Marine Lunven, ${ }^{6,7,8}$ Dominic Pérennou, ${ }^{9,10}$ Pascal Giraux, ${ }^{11,12}$ \\ Alexandre Foncelle, ${ }^{2}$ Yves Rossetti, ${ }^{1,2,3}$ Sophie Jacquin-Courtois, ${ }^{1,2,3}$ \\ Jacques Luauté, ${ }^{1,2,3}$ Gilles Rode ${ }^{1,2,3}$
}

To cite: Hugues A, GuinetLacoste A, Bin S, et al. Effects of prismatic adaptation on balance and postural disorders in patients with chronic right stroke: protocol for a multicentre double-blind randomised shamcontrolled trial. BMJ Open 2021;11:e052086. doi:10.1136/ bmjopen-2021-052086

- Prepublication history and additional supplemental material for this paper are available online. To view these files, please visit the journal online (http://dx.doi.org/10.1136/ bmjopen-2021-052086).

Received 10 April 2021 Accepted 21 October 2021

Check for updates

(C) Author(s) (or their employer(s)) 2021. Re-use permitted under CC BY-NC. No commercial re-use. See rights and permissions. Published by BMJ.

For numbered affiliations see end of article.

Correspondence to Aurelien Hugues;

huguesaurelien@gmail.com

\section{ABSTRACT}

Introduction Patients with right stroke lesion have postural and balance disorders, including weight-bearing asymmetry, more pronounced than patients with left stroke lesion. Spatial cognition disorders post-stroke, such as misperceptions of subjective straight-ahead and subjective longitudinal body axis, are suspected to be involved in these postural and balance disorders. Prismatic adaptation has showed beneficial effects to reduce visuomotor disorders but also an expansion of effects on cognitive functions, including spatial cognition. Preliminary studies with a low level of evidence have suggested positive effects of prismatic adaptation on weight-bearing asymmetry and balance after stroke. The objective is to investigate the effects of this intervention on balance but also on postural disorders, subjective straight-ahead, longitudinal body axis and autonomy in patients with chronic right stroke lesion.

Methods and analysis In this multicentre randomised double-blind sham-controlled trial, we will include 28 patients aged from 18 to 80 years, with a first right supratentorial stroke lesion at chronic stage ( $\geq 12$ months) and having a bearing $\geq 60 \%$ of body weight on the right lower limb. Participants will be randomly assigned to the experimental group (performing pointing tasks while wearing glasses shifting optical axis of 10 degrees towards the right side) or to the control group (performing the same procedure while wearing neutral glasses without optical deviation). All participants will receive a 20 min daily session for 2 weeks in addition to conventional rehabilitation. The primary outcome will be the balance measured using the Berg Balance Scale. Secondary outcomes will include weight-bearing asymmetry and parameters of body sway during static posturographic assessments, as well as lateropulsion (measured using the Scale for Contraversive Pushing), subjective straight-ahead, longitudinal body axis and autonomy (measured using the Barthel Index). Ethics and dissemination The study has been approved by the ethical review board in France. Findings will be submitted to peer-reviewed journals relative to rehabilitation or stroke.

\section{Strengths and limitations of this study}

- Evidence of short-term and long-term effects of prismatic adaptation (PA) on balance and postural disorders in adult patients with right chronic stroke.

- To contribute to the understanding of effects of PA but also the influence of spatial reference frames on balance and postural disorders in patients with stroke.

- Using a multicentre randomised sham-controlled trial with blinding of assessors and patients and intention-to-treat analyses.

- A cheap intervention, easy to implement, with no adverse event known, and not requiring a high level of active participation from patients.

Trial registration number NCT03154138.

\section{INTRODUCTION}

Background

Strokes frequently cause postural disorders, including a weight-bearing asymmetry (WBA) towards the non-paretic lower limb ${ }^{1-7}$ and a greater body sway ${ }^{26-9}$ during a standing posturographic assessment, but also an impaired body orientation with respect to gravity (known as lateropulsion) $\cdot{ }^{10-12}$ Patients also suffer from balance disorders ${ }^{9} 13 \quad 14$ limiting their level of activity and participation. ${ }^{15-18}$ They experience a greater number of falls. ${ }^{19-21}$ Gait and quality of life are both associated with balance, ${ }^{22-25}$ which underlines the importance of balance in patients who had a stroke.

After stroke, patients can also experience perturbations of spatial cognition. Between $32.5 \%$ and $63 \%$ of patients who had a stroke have a bias of subjective verticals ${ }^{1026-28}$ and a 
meta-analysis reported that the mean estimation of the subjective visual vertical was significantly deviated in patients who had a stroke compared with healthy participants. ${ }^{29}$ The longitudinal body axis (LBA) could be perceived with a deviation towards the contralesional side after stroke. ${ }^{30}$ The estimation of subjective straight-ahead (SSA) could also be shown as deviated and/or uncertain, especially in patients after right stroke lesion with an unilateral spatial neglect (USN) which perceived SSA rotated towards the ipsilesional side. ${ }^{31-34}$ These misperceptions of spatial reference frames were found more frequently and/or with higher magnitude after right stroke lesion than left stroke lesion..$^{102628303235}$ This could be the consequence of a predominance of the right hemisphere in spatial cognition, as for USN, which is more frequent, severe and persistent after a right stroke lesion than a left one in right-handed subjects. ${ }^{36}$ Furthermore, patients with right stroke lesion were displaying greater postural (ie, WBA, body sway, lateropulsion) and balance disorders. ${ }^{2} 51437$ Taking into account that the misperceptions of spatial reference frames such as the subjective (visual or postural) vertical and the LBA were found associated with body sway, ${ }^{26}$ lateropulsion, ${ }^{10}{ }^{12} \mathrm{WBA}^{38}$ and balance, ${ }^{26}$ the postural and balance disorders after stroke are strongly suspected to be influenced by spatial cognition disorders. Although considered by some authors as a potential strategy of compensation to ensure a better stability, ${ }^{39-41}$ the underlying mechanisms of WBA partly involve egocentric spatial reference frames. ${ }^{38}$

\section{State of the art}

The rehabilitation of balance is a common goal for patients with stroke. Nowadays, few rehabilitations are considered as effective. ${ }^{42}$ Among these is task-oriented training, which involves practicing functional, specific and goal-centred tasks based on motor learning principles such as repetition, variability or feedback. ${ }^{43} 44$ Due to a potential representational origin to balance and postural disorders, using techniques which modulate spatial reference frames, such as prismatic adaptation (PA) the effect of which to USN is well known, ${ }^{3145-47}$ could be relevant. In addition, PA would be suitable in patients with severe impairments or having deficits of attention and behavioural disorders.

PA is an intervention consisting in repetitive pointing tasks while the patient is wearing glasses shifting the optical axis towards the right. Under prism exposure, first pointing movements are deviated toward the right side as patients are not able to point at the target. These errors are compensated for once they are noticed and successive trials are performed. When subjects are asked to point straight-ahead after removal of prisms, a shift opposite to the prism deviation is observed, reflecting a 'true' adaptation also called sensorimotor after-effect. ${ }^{31} 48$ This individuals' behaviour during PA could be explained by two successive mechanisms: A process of recalibration which is a compensatory response needed to modify the motor commands; then a spatial realignment needed to align conflicting visuomotor and proprioceptive-motor reference frames. ${ }^{49-52}$ Very interestingly, numerous studies in healthy subjects and brain-injured patients showed expansion of the sensorimotor after-effects of PA to unexposed sensory, motor and cognitive functions, such as spatial cognition. ${ }^{31}{ }^{45} 53-55$ A 'bottom-up' processing of information from peripheral sensorimotor inputs to high-level cognitive centres bypassing the patient's awareness may explain the expansion of sensorimotor after-effect to cognitive post-effects. ${ }^{31456}$ The involvement of cerebelloparietal network in the sensorimotor after-effect and the 'bottom-up' activation of prefrontal and temporal areas for cognitive post-effects are strongly suspected. ${ }^{52}$ 57-59 PA effects could vary according to neuroanatomical individual features. Patients with greater PA-induced cognitive effects on USN showed a significant contribution of undamaged hemisphere and interhemispheric connections. ${ }^{60}$

Regarding postural and balance disorders, five studies ${ }^{61-65}$ have found a significant reduction in WBA (in sitting or standing position) after using PA with an optical deviation of 10 degrees towards the right in patients with acute or chronic right stroke lesion. However, only one of them assessed effects of PA on balance. Hugues et al have found an improvement of balance after PA in patients at chronic stage without USN jointly with a significant left shift of SSA, a significant reduction in WBA and lateropulsion. ${ }^{62}$ This study did not include a control group and the efficacy of PA on balance disorders after stroke is not yet evidenced. To our knowledge, one randomised controlled trial investigating the effects of PA on postural disorders in patients with a right stroke lesion is ongoing, and results are not yet published. This trial enrols patients with stroke at an acute or subacute stage and compares the effects of neck muscle vibrations, PA, conventional rehabilitation or both PA and neck muscle vibrations on WBA as primary outcome (register number: NCT01677091). To our knowledge, no study has investigated the effects of PA on balance compared with sham intervention in patients with a chronic right stroke.

\section{Objectives and hypothesis}

The aim of the present study is to investigate efficacy of 2 weeks of PA on balance as primary outcome, but also on postural disorders, autonomy and egocentric spatial reference frames (assessed by SSA and LBA) as secondary outcomes, compared with sham intervention on patients with right stroke at chronic stage. A secondary objective is to investigate the relationship between PA-induced changes on misperceptions of spatial reference frames and these on postural and balance disorders. We hypothesise that PA would improve the balance of patients with a right chronic stroke lesion jointly with a reduction in postural disorders, resulting from a 'bottom-up' effect of PA on egocentric spatial reference frames.

Additional objectives are to determine brain lesions involved in misperceptions of spatial reference frames, postural and balance disorders; and to assess the 
relationship between brain lesions and PA-induced changes on performances.

\section{METHODS}

The protocol (sixth version from 12 December 2019) was developed using the Standard Protocol Items: Recommendations for Interventional Trials statement (online supplemental material). ${ }^{66}$

\section{Design}

This is a prospective multicentre randomised doubleblind sham-controlled superiority trial conducted in three units of physical and rehabilitation medicine in France (Hospices Civils de Lyon; Centre Hospitalier Universitaire Grenoble-Alpes; Centre Hospitalier Universitaire de Saint-Etienne). Participants will be randomised in two parallel groups: the experimental group will receive 10 daily sessions of $\mathrm{PA}$ while the control group will receive 10 daily sessions of sham intervention. In addition, all participants will receive conventional rehabilitation regardless of the allocation group.

\section{Participants and criteria of inclusion}

The inclusion criteria are: (1) being aged from 18 to 80 years; (2) having a first right unilateral supratentorial, ischaemic or haemorrhagic, stroke as defined by the WHO and diagnosed on the basis on both a clinical examination and a CT or MRI scan confirmation; (3) having had a stroke more than a year ago (time poststroke $\geq 12$ months); (4) being able to stand for at least $30 \mathrm{~s}$ with eyes open and with eyes closed; (5) having a bearing $\geq 60 \%$ of body weight on the non-paretic lower limb during a posturographic assessment in quiet static standing position with eyes open; and (6) signing an informed and free consent. Loading less than $40 \%$ of body weight on the paretic lower limb was suggested to be a target in rehabilitation, as reflecting a level of postural disorders likely to limit gait abilities $(95 \%$ of patients with a such WBA after stroke were unable to walk without a standard cane) ${ }^{67}$ The exclusion criteria are: (1) having a brainstem, cerebellum or bilateral stroke lesion; (2) having any orthopaedic or rheumatism disease, visual deficit due to a retina disease or any other disease likely to interfere with the assessments of the study according to the judgement of investigators; (3) having any difficulty to speak or understand the language or psychiatric disorders limiting the understanding of the instructions, the procedures and the consent collection; and (4) being pregnant or breast feeding, being subject of a guardianship or tutelage measure or not having social health insurance. The study started in December 2017, and the end date is planned for 2023 .

\section{Procedure}

A standardised procedure will be conducted in all the participating centres (figure 1). Patients with stroke admitted in rehabilitation units or coming for their ongoing medical follow-up will be screened. Patients who volunteer to participate will then meet with a medical physician authorised by the study for an inclusion visit in order to formally check the inclusion/exclusion criteria and to collect their informed consent. The study duration for each participant will be of 3 months and 3 weeks (111 days). During the first week, patient characteristics at baseline will be collected and two pre-intervention assessments will be performed on two different days. Then, the randomisation will take place. A structural brain MRI will also be carried out preferably during the first week. During the second and third weeks, participants will receive PA or sham intervention according to their allocation group, 5 days per week. The day of the last session of PA/sham will be considered as 'day 0' (D0). In order to determine immediate (sensorimotor) and delayed (by expansion to unexposed functions) effects, ${ }^{45} 5262$ the post-intervention assessments will be performed 2 hours after the last $\mathrm{PA} /$ sham session, and 3 and 7 days after D0. Following these 4 weeks in units of physical and rehabilitation medicine, participants will either be discharged from the hospital, or continue their hospital stay according to their clinical needs. Participants will also be assessed 1 and 3 months after $\mathrm{D} 0$, in order to assess potential sustained effects.

\section{Randomisation}

The randomisation will be stratified according to the centres and the presence of USN (yes/no) determined using the GEREN test battery (French Collaborative Study Group on Assessment of USN; see outcome part below) ${ }^{68}$ A computer-generated randomisation list with blocks and a 1:1 ratio will be used for group allocation (SAS V.9.3, SAS Institute). This list will be managed and stored by only two independent investigators (SB and LV) from outside the units where the study will take place. The list will be not accessible to any other investigators, staff members or patients. The randomisation will be performed after the completion of baseline and pre-intervention assessments. Because SB or LV will not be involved in any part of the screening, eligibility or inclusion of participants, the allocation will be concealed.

\section{Interventions}

The therapists in charge of PA/sham intervention will be trained before the opening of the centre. Only these therapists will be informed of the allocation group, neither the participant nor any staff member will be aware of it. The PA or sham interventions will be delivered in a room separated from other rehabilitation settings, and will be the same for the two groups. Apart from these having a high technical knowledge of PA, patients are not likely to identify which glasses are used. As patients frequently have cognitive deficits after stroke, their ability to discover the allocation group 


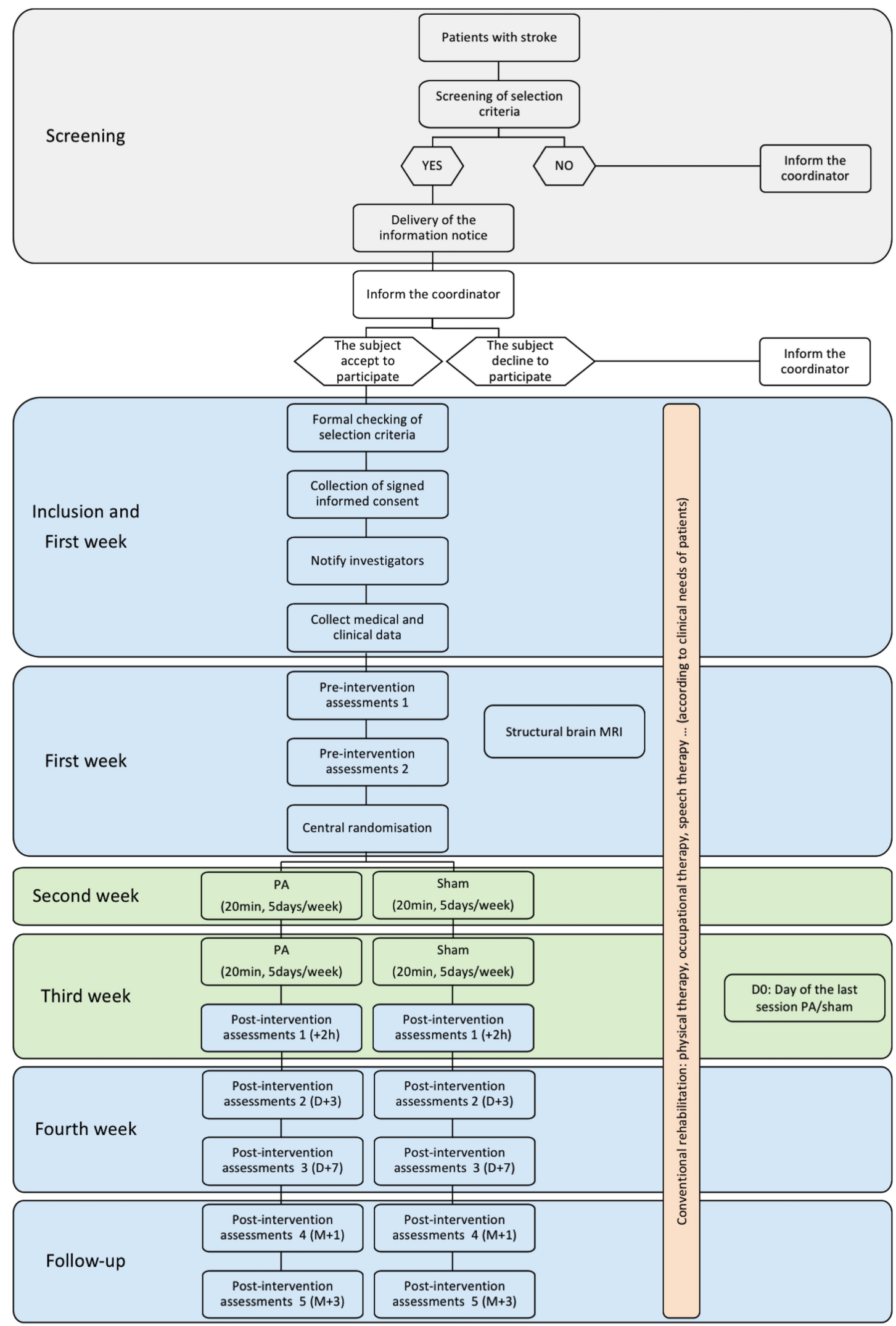

Figure 1 Procedure from enrolment to the end of study. PA, prismatic adaptation.

appears very weak and we can therefore consider the patient blinding as sufficient.

No change of intervention group will be allowed. If this were to happen, the therapists should correct deviations for the remaining sessions. Intention-to-treat analyses will be primarily performed. In case of any medical event leading to a worsening of the patient's health condition or that may modify his balance and/or 
A

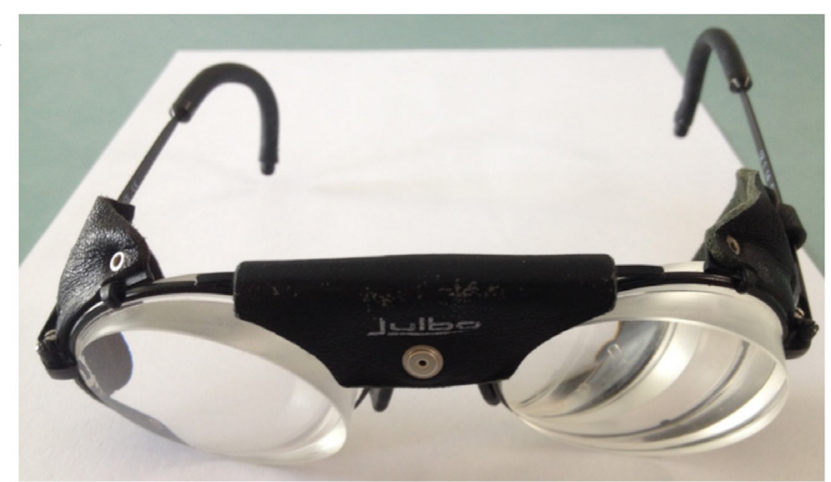

B

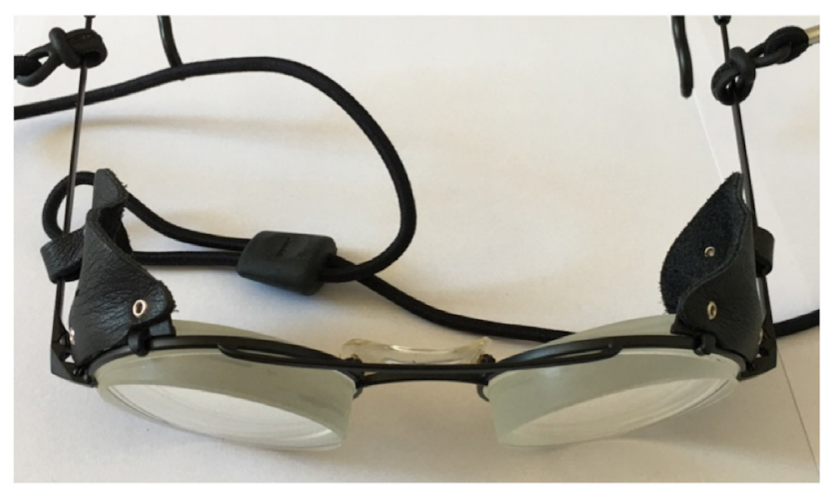

Figure 2 Prismatic glasses (A) and sham glasses (B). (A) A pair of prismatic glasses with an optical deviation of 10 degrees towards to the right side; (B) a pair of sham glasses with a neutral optical deviation.

postural function, the investigators could decide to stop the participation of the subject in the trial.

\section{Prism adaptation}

According to the method previously used, ${ }^{31464761}$ the participant will wear prim glasses (OptiquePeter.com) shifting the optical axis of 10 degrees towards the right side (figure 2). During $20 \mathrm{~min}$ prism exposure, the therapist will pseudo-randomly ask the patient to make several sets of approximately 50 rapid pointing movements towards two different visual targets positioned 10 degrees on the right and left sides of the midline body axis of the patient. During the first pointing tasks, the patient's movement is shifted towards the right side of the visual target. Then, the participant will take into account the initial error and will compensate it in order to reach the target. At the end of the PA session, when a pointing movement is asked of the patient after having removed the glasses, this one will be deviated towards the left side of the target. This constitutes the visuomotor (sensorimotor) 'after-effect' of PA (figure 3). ${ }^{3146}$ The PA will be dispensed daily in $20 \mathrm{~min}$ sessions, 5 days per week during 2 weeks. If a session is missed, two sessions could be carried out the following day. Thirty minutes per session will be scheduled to take into account the time for installation, explanations and potential rests. No adverse event has ever been reported before. ${ }^{31464761}$

\section{Sham intervention}

The procedures and the modalities will be identical to those applied in the experimental group except for the glasses used. The device will be fitted out two prismatic lenses set up so as not to deviate the optical axis (figure 2). The sham glasses will look like prismatic glasses.

\section{Conventional rehabilitation}

In addition to PA or sham intervention, the participants will receive a conventional rehabilitation (physical therapy, occupational therapy or speech therapy) not exceeding $90 \mathrm{~min}$ on average per day. The content and the duration will be determined by the physician of the unit in charge of the participant according to the clinical needs and before the beginning of the study. Therapists in charge of the conventional rehabilitation should not know the allocation group of participants. Rehabilitations likely to modify spatial cognition such as biofeedback platform, virtual reality, other sensory interventions or constraint-induced therapy, will be proscribed during the duration of the study.

\section{Outcomes}

All assessors will be blinded to the allocation group and they cannot be in charge of conventional rehabilitation. Data extracted from medical records will be: age in years, sex, manual laterality measured using the Edinburgh Handedness Inventory, time post-stroke in days, the type (ischaemia or haemorrhage) and the location of the stroke lesion based on recent CT or MRI scans, medical history, the presence of an aphasia and lateral homonymous hemianopia and current medication. In addition, we will assess at baseline motor weakness but also spasticity, and superficial and deep sensibility of left body (online supplemental material). The presence of USN will be determined using GEREN tests. ${ }^{68}$ This battery of tests includes (1) a preliminary assessment of awareness, sensorial extinction and hemianopia, (2) an assessment of gaze orientation and personal neglect, (3) an assessment of extrapersonal neglect using paper and pencil tests (the bells test, figure copying, clock drawing, the line bisection, the overlapping figures test, a reading test and a writing test) and (4) a behavioural assessment of neglect and anosognosia using the Catherine Bergego Scale. Based on the cut-off defining normality or abnormality for each test, we will consider the presence of USN if at least one test is abnormal. Overall cognitive disorders will be measured using the Mini-Mental State Examination.

The duration and frequency of interventions during the first 4 weeks after inclusion, as well as the observance and compliance of patients to $\mathrm{PA} /$ sham sessions will be monitored. The summary of assessments at each time point is reported in table 1 .

\section{Primary outcome}

Based on published results suggesting a significant positive effect 7 days after the last PA session, ${ }^{62}$ the primary outcome will be the balance measured at $\mathrm{D}+7$ using the 


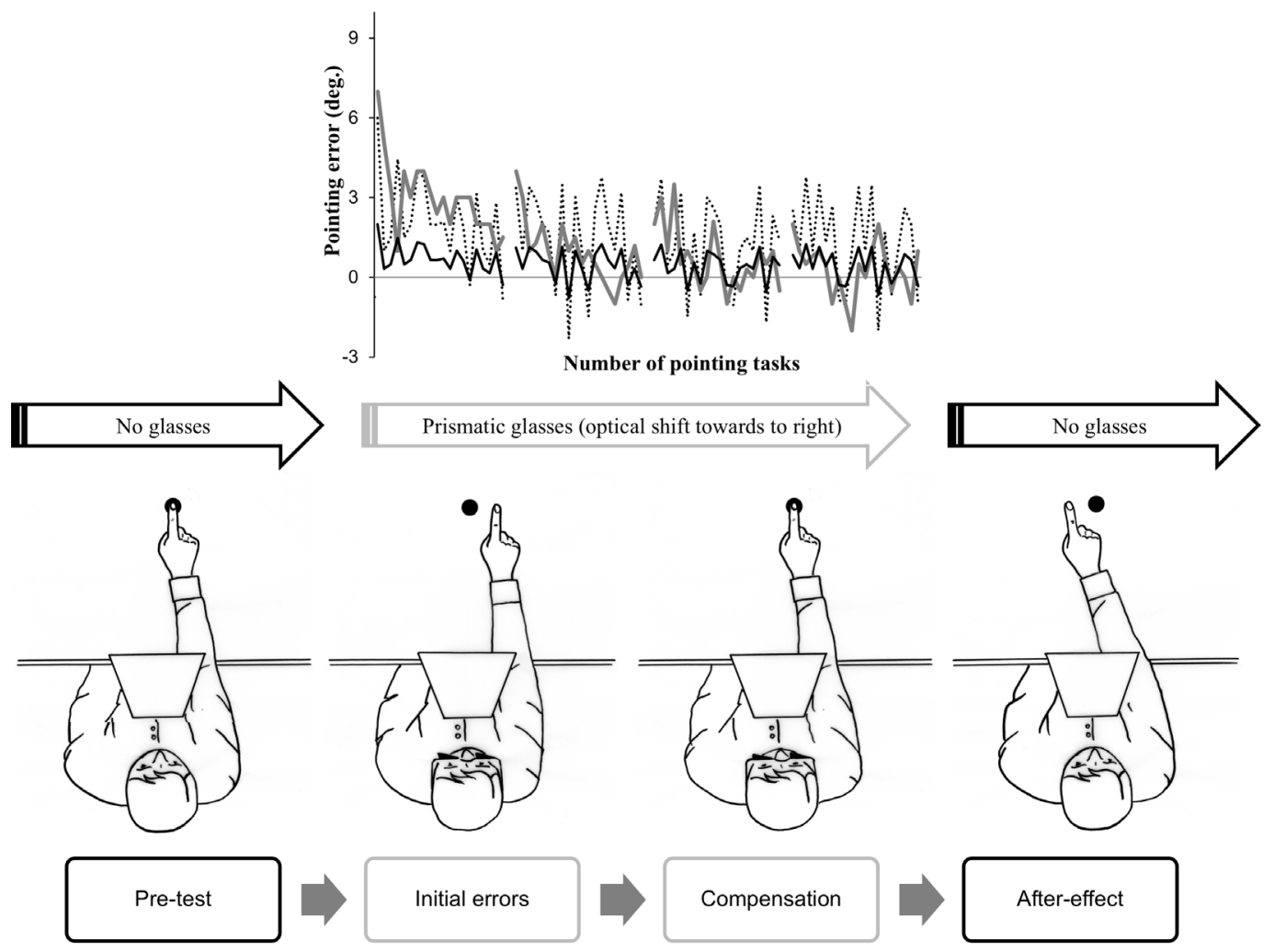

Figure 3 Procedure for prismatic adaptation. The participant will be seated in front of the support set up on a table, with the chin on a part of the support limiting the inclination or rotation of the head and placed in the midline body axis. To limit visuo-feedback during pointing tasks with prism exposure, the support hides the initial position of the patient's hand but also at the beginning of the movement course (ie, 20\%-50\%). In addition, the therapist will ensure that the patient perform rapid movements (adapted from Rode et al., 2015).

Berg Balance Scale (BBS). Validated in patients with stroke, ${ }^{69}$ this scale comprises 14 items each scored from 0 to 4 and assesses the functional abilities of balance (the higher the score, the better the balance).

\section{Secondary outcomes}

The secondary outcomes will be: the balance assessed using the BBS at $\mathrm{M}+1$ and $\mathrm{M}+3$; postural disorders assessed at +2 -hour, $\mathrm{D}+3, \mathrm{D}+7, \mathrm{M}+1$ and $\mathrm{M}+3$ (the mean mediolateral position of centre of pressure $(\mathrm{COP} ; \mathrm{mm})$, the mean anteroposterior position of COP $(\mathrm{mm})$, the sway area of COP $\left(\mathrm{mm}^{2}\right)$, the SD of mediolateral position of COP, the SD of anteroposterior position of COP and body weight bearing on left and right lower limb (per cent of body weight) measured during a posturographic evaluation, but also lateropulsion measured using the Scale for Contraversive Pushing (SCP)); egocentric spatial reference frames measured at +2 -hour, $\mathrm{D}+3, \mathrm{D}+7$, $\mathrm{M}+1$ and $\mathrm{M}+3$ using manual SSA, visual SSA, open-loop pointing (OLP) and LBA; and autonomy measured at $\mathrm{D}+7, \mathrm{M}+1$ and $\mathrm{M}+3$ using the Barthel Index.

In standing static position, the posturographic assessment measures the spatial and temporal evolution of the COP by means of two separate force platforms, one under each foot, parallel and $14 \mathrm{~cm}$ spaced (Freetest 6 , Technoconcept). Two trials will be performed with eyes opened and two others with eyes closed, each trial will be recorded for $30 \mathrm{~s}$. The mean of two tests for each eye condition will be calculated. No human or technical help during measurements will be allowed. As its reliability is considered as acceptable, posturographic assessment is frequently implemented to assess postural disorders after stroke. $^{7071}$

The SCP is composed of three parts and assesses: the symmetry of the spontaneous posture, the extension of the area of physical contact to the ground by using an arm or leg and the resistance to passive correction of posture to an upright position. Both standing and sitting positions are assessed and each contributes to $50 \%$ of the score of each part (between 0 and 2 points). On a total of 6 points, a participant with a score $\leq 0.5$ is considered as 'upright' while a participant with a score $>0.5$ is considered as having a lateropulsion. A contraversive pushing is considered if the score reaches at least 1 for each of three parts of SCP. ${ }^{1072}$

The SSA assessment will be performed in accordance with the method used in Rossetti $e t a l^{31}$ and in Rode $e t a l^{46}$ (figure 4). The SSA corresponds to the perception of the sagittal axis in the horizontal plan and thus in the egocentric peripersonal space. Seated in front of the device, the patient will indicate his/her subjective 'straight-ahead' 
Table 1 Summary of baseline, pre-intervention and post-intervention assessments at each time point

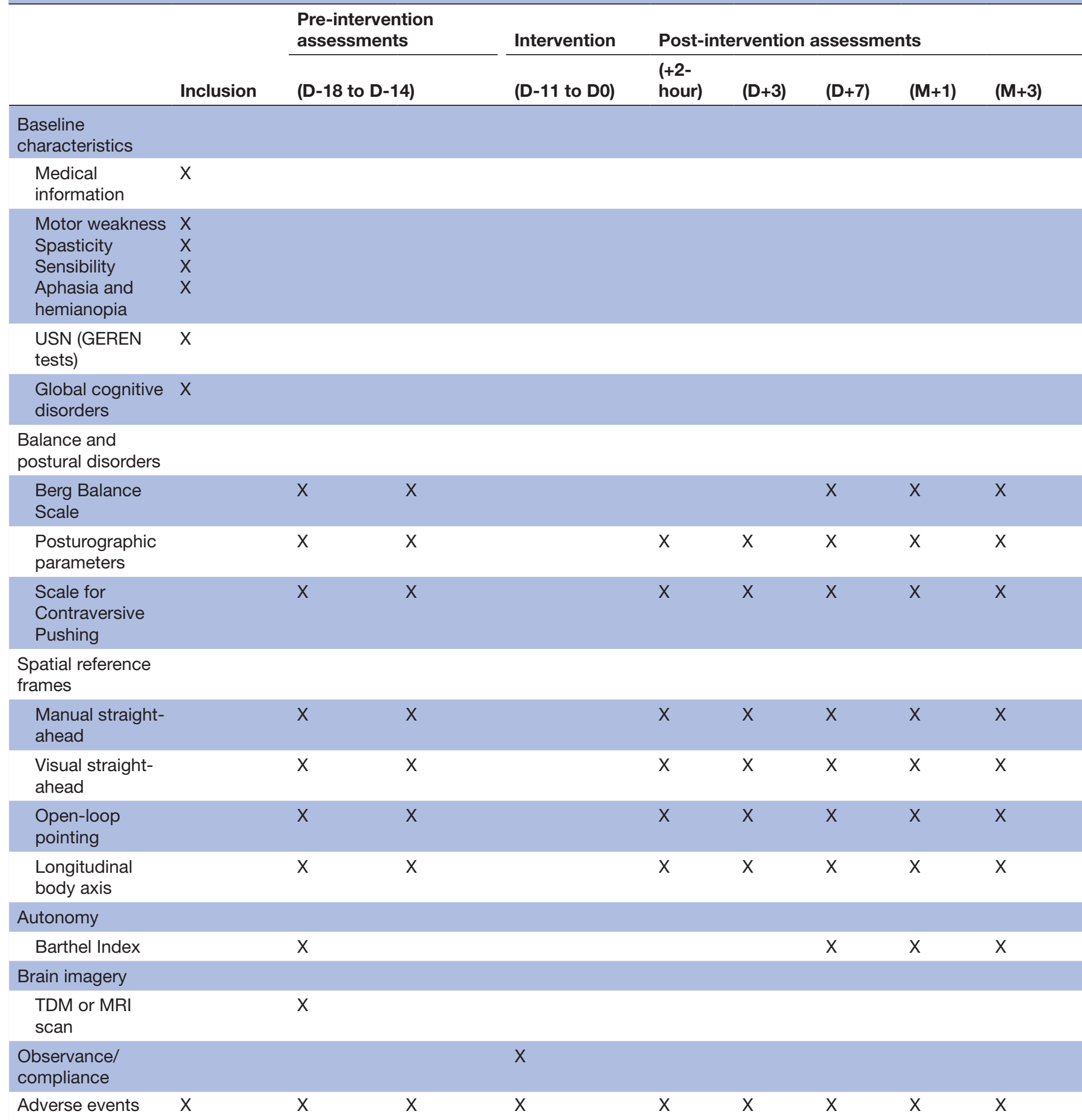

D, day; GEREN, French Collaborative Study Group on Assessment of Unilateral Spatial Neglect; H, hour; M, month; MRI, Magnetic resonance imaging; TDM, tomodensitometry; USN, unilateral spatial neglect.

direction by a pointing task with a finger of the right hand without visual input for manual SSA. For visual SSA, the patient is seating in the dark and will be asked to say when the luminescent diode moving in front of him/her is perceived in a 'straight-ahead' position. The manual SSA therefore reflects the proprioceptive modality while the visual SSA reflects the visual modality. For OLP, the patient will be asked to point with a finger of the right hand at the target drip-line as precisely as possible without time constraint. The pre-intervention and postintervention test difference on OLP indicates the magnitude of the PA total effect. For each test, 10 trials will be performed.

The LBA corresponds to the patient's representation of the personal egocentric space. Here, the procedure of LBA assessment is similar to the one used in Barra et al. ${ }^{30} 38$ 

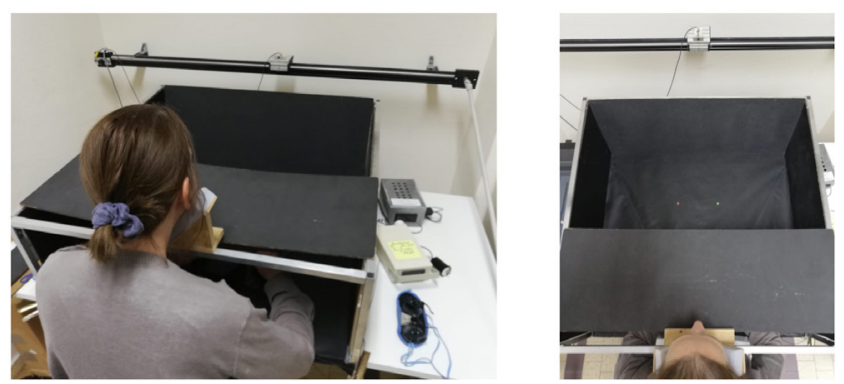

Figure 4 Subjective straight-ahead assessment. The patient is seated in front of the device set up on a table, with the chin on a part of the device preventing the inclination or rotation of the head. The midline device axis will match the patient sagittal axis. For the manual SSA, the assessor asks the patient, placed in the dark, to point on the horizontal plan of the device with the forefinger of the right hand the 'straightahead' direction. From a departure position of the right hand closer to navel, the patient spreads the arm without restriction, then returns to the initial position. The pointing is measured by means of an electronic system included in the horizontal plan of the device. The angular deviation from the objective sagittal axis is displayed by the device. For the visual SSA, the patient keeps the initial position of manual SSA and the measurement is still performed in a total darkness. A luminescent red diode will move in front of the patient from the extreme left or right position in the visual field towards the opposite extreme position at a slow speed. The diode is at the same height than the gaze of the patient. The head of the patient is still contained in the chin support limiting its inclination and rotation. The investigator asks the patient to say stop when the red diode reaches the position perceived as being 'straight-ahead'. The angular deviation from the objective sagittal axis is also displayed by the device. For the open-loop pointing, the patient is still in total darkness and takes the initial position of manual SSA with his/her hand closer to his/her navel. The red luminescent diode is aligned with the objective sagittal axis of the patient. The investigator asks the patient to point in the direction of the red diode on the horizontal plan of the device with the forefinger of the right hand, and then to return to the initial position. For each test, 10 trials will be performed. For visual SSA, five trials will be performed with a departure position of the red diode on the right side of the patient and five others on the left side. SSA, subjective straight-ahead.

Patients will be lying on an examination table in a total darkness with their head, trunk and lower limbs aligned by the assessor. The head and the lower limbs will be laterally constrained. Placed at approximately $25-30 \mathrm{~cm}$ above the patient, a device containing a fluorescent tube will be moved by the assessor from the extreme left or right position in the patients' visual field towards the opposite extreme position at a slow speed. Each patient will be asked to say stop when the fluorescent tube reaches the position perceived as being overlapping with his/ her LBA. Five trials will be performed with a departure position of the fluorescent tube on the right side and five others on the left side. Between each trial, patients will have to close their eyes to prevent any visual feedback or cueing.
For SSA and LBA, the average deviation will be determined using the mean of 10 trials and the uncertainty in the estimate using the SD of 10 trials. By convention, a negative value indicates a deviation towards the left side and a positive value towards the right side.

The Barthel Index is a 10-item scale widely used to assess the functional independence in daily-living activities. A maximal score of 100 points indicates total independence. Its metrological properties are considered as good, which makes it relevant and appropriate to assess autonomy in patients with stroke. ${ }^{73}$

All participants will undergo a brain structural MRI scan. Lesions will be manually delineated on native-space T1-weighted images for each patient. T1-weighted images will be normalised to the template MNI152 using affine and diffeomorphic deformations ${ }^{74}$ implemented in BCBtoolkit $^{76}$ (http://www.toolkit.bcblab.com). Finally, lesions will be manually drawn in the MNI space. Subsequently, lesions will be overlapped to highlight damaged areas using MRICron. ${ }^{77}$ A voxel-based lesion-symptom mapping (VLSM) analysis will be performed using the non-parametric rank-order Brunner-Munzel analysis with voxel-based permutation (1000; http://www.cabiatl.com/ mrico/npm/). ${ }^{77}$ The VLSM analysis will be run for the dependent continuous variable of interest and controlled for the overall lesion size. Only voxels damaged in at least $10 \%$ of patients will be included in the analysis. Results will be projected onto a high-resolution template in standard space. For atlas-based mapping of white matter disconnection, we will map the lesion from each patient onto tractography reconstructions of white matter pathways obtained from a group of healthy controls. ${ }^{78}$ We will quantify the severity of the disconnection by measuring the probability of the tract to be disconnected ${ }^{79}$ using the Tractotron software as part of the BCBtoolkit. ${ }^{76}$ In the resulting disconnectome map, the voxels will show the probability of disconnection from $0 \%$ to $100 \%$. Statistical analyses (association with clinical disorders and PA-induced changes) will be performed on these maps using the 'Randomise' function implemented in FSL (https:/ / fsl.fmrib.ox.ac.uk/fsl/fslwiki/FSL), with 5000 random permutation tests and a Threshold-Free Cluster Enhancement option. Results will be adjusted for family-wise error corrections for multiple comparisons. In accordance with the additional objectives, we will perform regressions between the grey and white matter brain damages and (i) misperceptions of spatial reference frames, postural and balance disorders; and (ii) the changes induced by PA on these outcomes.

\section{Statistical analysis}

Descriptive statistics will be computed using count (percentage), mean $\pm \mathrm{SD}$ or median (IQR) as appropriate. We will compare baseline characteristics between groups using the Fisher's exact test or the Pearson's $\chi^{2}$ test for qualitative variables, and the independent t-test for continuous measures (or Mann-Whitney test if the hypothesis of normal distribution is rejected). 
Unadjusted means and SEs will be estimated for the primary and secondary continuous outcomes at each time point. Then, we will build linear mixed-effect models with time as within-participant factor, group as betweenparticipant factor and time by group interaction. The centres and USN will be included as covariates. Group, time and USN will be considered as fixed factors, while participants and centres as random factors ( $\mathrm{R}$ package 'nlme'). The group by time interaction will be examined as well as models including a random intercept only and a random intercept and slopes. The different models will be compared using Akaike and Bayesian information criterion and analysis of residuals. In case of significant effect, we will perform multiple comparisons adjusted using the Bonferroni-like method according to the objectives previously stated. When two pre-intervention assessments will have be done, the mean and the individual values of assessments will be used. Based on previous studies, we expect that the BBS variable will follow a normal distribution or a Poisson distribution, making necessary the use of a mixed generalised linear model. Otherwise, we could implement data transformations or non-parametric tests.

We will carry out intention-to-treat analyses. Missing data will be handled by mixed-effects models. For secondary outcomes, per-protocol analyses will be additionally computed. We will also perform sensitivity analyses using an analysis of covariance controlling for baseline values as fixed factor. ${ }^{80-82}$ Additional planned analyses are reported in online supplemental material.

The investigator in charge of analyses will be blinded to allocation group. All statistical analyses will be performed using R software (R Foundation for Statistical Computing, Vienna, Austria; available in http://www.R-project.org/) with a $p$ value $\leq 0.05$ considered as statistically significant.

\section{Sample size}

According to the pilot study, ${ }^{62}$ we could postulate a within-group difference at $\mathrm{D}+7$ of 4.83 points on BBS for the experimental group and of 2 points for the control group, with a pooled SD of change of 2 . To our best knowledge, in patients after stroke at chronic stage, the minimal clinically important difference for BBS was never assessed while the minimal detectable change was estimated between 2.5 and 4.7 points. ${ }^{83-86}$ On the basis of these hypotheses, considering alpha risk of 0.05 , a statistical power of $95 \%$, and a bilateral test, we have to include 13 participants per group. To take into account potential lost to follow-up or dropping out before the primary outcome, one additional participant by group could be included. We could therefore include 28 participants.

\section{Ethics and dissemination}

Promoted by the Hospices Civils de Lyon, this study was approved by an institutional review board ('Comité de protection des personnes Nord Ouest IV'; 2017-A0180944) and registered on ClinicalTrials.gov. The procedure will be performed in respect of the Helsinki Declaration. Before inclusion, information relative to the study will be given to eligible participants. The participants agreeing to participate will sign a free and informed consent. Any potential important modification of protocol will be submitted to the institutional review board according to the French law. Procedures to collect and store patient data are in accordance with General Data Protection Regulation of European Union, and have been declared to the Commission nationale de l'informatique et des libertés (CNIL: French committee for data protection) in accordance with existing regulations in France. Patients will not have to support any cost related to their participation in the study. They remain free to discontinue their participation in the trial. All participants will receive conventional rehabilitation in accordance with current practices. The risk for participants is considered as low: PA is frequently used to treat USN and, to our knowledge no adverse event has ever been reported.

Information relative to data management and monitoring are reported in online supplemental material.

\section{Patient and public involvement, and dissemination}

The protocol presented herein was developed without patient or public involvement. Neither participants nor the public will not be involved in result analysis and their interpretation, as well as in the writing of the final manuscript. The results will be submitted to peer-reviewed journals relative to rehabilitation or stroke, as well as to international congresses, in order to disseminate findings and discuss the interest of PA in rehabilitation with researchers, healthcare givers and patients. Authorship eligibility will be based on the criteria of the International Committee of Medical Journal Editors. The full protocol (in French), the model consent form (in French), the data that will support the study results and the statistical code will be available from on reasonable request to the corresponding author and GR.

\section{DISCUSSION}

The interest of the study presented herein is to determine if PA could improve balance and postural disorders in patients with right stroke lesion at a chronic stage. By a bottom-up action from a sensorimotor representation level to a cognitive representation level, PA is likely to modify postural disorders related to misperceptions of spatial reference frames. The present study is a pragmatic trial focusing on balance as the primary outcome. Considered as a level of activity in the model of disability according to International Classification of Functioning, Disability and Health, balance is an essential concern for patients after stroke. The use of a multicentre randomised sham-controlled trial with blinding of assessors and patients and intention-to-treat analyses is consistent with international scientific standards to assess the efficacy of interventions. ${ }^{87}$

PA is thought to have many advantages: it is cheap and easy to implement with only one therapist; its use is safe as no adverse event has been reported although it is used 
in clinical practice to treat USN; it can be delivered to patients both in acute-subacute or chronic stage, with light or severe impairments, and to inpatients, outpatients and at home. In addition, as it does not require a high level of participation from patients, PA could be a relevant intervention in patients with severe attention deficits and could complement the range of existing interventions.

This trial also includes secondary explicative objectives which could contribute to improve the understanding of PA mechanisms, especially how the expansion of sensorimotor after-effects of PA on spatial references frames could improve balance. This could be of high relevance from a theoretical and pragmatic point of view. In addition, the neuroanatomical study could contribute to identify patients likely to be responders by highlighting neural networks likely to mediate effects of PA.

\section{Author affiliations}

${ }^{1}$ Service de médecine physique et réadaptation, hôpital Henry Gabrielle, Hospices Civils de Lyon, Saint-Genis Laval, France

${ }^{2}$ Equipe 'Trajectoires', Centre de Recherche en Neurosciences de Lyon, Inserm UMR-S 1028, CNRS UMR 5292, Université de Lyon, Université Lyon 1, Bron, France ${ }^{3}$ Plate-forme 'Mouvement et Handicap', hôpital Henry Gabrielle, Hospices Civils de Lyon, Saint-Genis-Laval, France

${ }^{4}$ Service de Recherche Clinique et Epidémiologique, Pôle de Santé Publique, Hospices Civils de Lyon, Lyon, France

${ }^{5}$ EMR 3738, Université Lyon 1, Villeurbanne, France

${ }^{6}$ Département d'Etudes Cognitives, École normale supérieure, PSL University, Paris, France

${ }^{7}$ Hôpital Henri Mondor-Albert Chenevier, Centre de référence Maladie de Huntington, Service de Neurologie, AP-HP, Créteil, France

${ }^{8}$ INSERM U955, Institut Mondor de Recherche Biomédicale, Equipe NeuroPsychologie Interventionnelle, Université Paris Est Créteil, Créteil, France ${ }^{9}$ Département de médecine physique et de réadaptation, Institut de rééducation, Hôpital sud, Centre Hospitalier Universitaire Grenoble-Alpes, Grenoble, France ${ }^{10}$ Laboratoire Neurosciences Cognitives, CNRS UMR5105, Université Grenoble Alpes, Grenoble, France

${ }^{11}$ Service de médecine physique et réadaptation, Hôpital Bellevue, Centre Hospitalier Universitaire de Saint-Etienne, Saint-Etienne, France

${ }^{12}$ Laboratoire Inter-universitaire de Biologie de la Motricité (LIBM, EA 7424), Université Jean Monnet Saint-Etienne, Université de Lyon, Saint-Etienne, France

\section{Twitter Aurélien Hugues @A_Hugues}

Acknowledgements The authors thank Hélène Boyer (Direction de la Recherche Clinique et de I'Innovation, Hospices Civils de Lyon) for help in manuscript preparation, and Lisette Arnesault for her contribution

Contributors Authorship eligibility will be based on the criteria of the International Committee of Medical Journal Editors (ICJME). All authors (AH, AG-L, SB, LV, ML, DP, PG, AF, YR, SJ-C, JL and GR) have contributed to the conception and the methodology of this protocol study. AH and GR were in charge of writing the original draft. The other authors (AG-L, SB, LV, ML, DP, PG, AF, YR, SJ-C and JL) have contributed to the reviewing and editing of the manuscript.

Funding This work was supported by the French health ministry ('Programme Hospitalier pour la Recherche Clinique Inter-régional', grant number PHRCI16-003). The funders had no role in the conception or the implementation of the research, the analysis or the interpretation of data and the writing or the review of the manuscript.

Competing interests None declared.

Patient consent for publication Not applicable.

Provenance and peer review Not commissioned; externally peer reviewed.

Data availability statement Data are available upon reasonable request.
Supplemental material This content has been supplied by the author(s). It has not been vetted by BMJ Publishing Group Limited (BMJ) and may not have been peer-reviewed. Any opinions or recommendations discussed are solely those of the author(s) and are not endorsed by BMJ. BMJ disclaims all liability and responsibility arising from any reliance placed on the content. Where the content includes any translated material, BMJ does not warrant the accuracy and reliability of the translations (including but not limited to local regulations, clinical guidelines, terminology, drug names and drug dosages), and is not responsible for any error and/or omissions arising from translation and adaptation or otherwise.

Open access This is an open access article distributed in accordance with the Creative Commons Attribution Non Commercial (CC BY-NC 4.0) license, which permits others to distribute, remix, adapt, build upon this work non-commercially, and license their derivative works on different terms, provided the original work is properly cited, appropriate credit is given, any changes made indicated, and the use is non-commercial. See: http://creativecommons.org/licenses/by-nc/4.0/.

ORCID iD

Aurélien Hugues http://orcid.org/0000-0001-9190-2867

\section{REFERENCES}

1 Sackley CM. The relationships between weight-bearing asymmetry after stroke, motor function and activities of daily living. Physiother Theory Pract 1990;6:179-85.

2 Rode G, Tiliket C, Boisson D. Predominance of postural imbalance in left hemiparetic patients. Scand J Rehabil Med 1997;29:11-16.

3 Geurts ACH, de Haart M, van Nes IJW, et al. A review of standing balance recovery from stroke. Gait Posture 2005;22:267-81.

4 Genthon N, Rougier P, Gissot A-S, et al. Contribution of each lower limb to upright standing in stroke patients. Stroke 2008;39:1793-9.

5 Ishii F, Matsukawa N, Horiba M, et al. Impaired ability to shift weight onto the non-paretic leg in right-cortical brain-damaged patients. Clin Neurol Neurosurg 2010;112:406-12.

6 Cheng PT, Liaw MY, Wong MK, et al. The sit-to-stand movement in stroke patients and its correlation with falling. Arch Phys Med Rehabil 1998;79:1043-6.

7 Pérennou D, Pélissier J, Amblard B. La posture et Le contrôle postural Du patient cérébrolésé vasculaire: une revue de la littérature. Annales de Réadaptation et de Médecine Physique 1996;39:497-513.

8 Dickstein R, Abulaffio N. Postural sway of the affected and nonaffected pelvis and leg in stance of hemiparetic patients. Arch Phys Med Rehabil 2000;81:364-7.

9 Portnoy S, Reif S, Mendelboim T, et al. Postural control of individuals with chronic stroke compared to healthy participants: Timed-Upand-Go, functional reach test and center of pressure movement. Eur J Phys Rehabil Med 2017;53:685-93.

10 Pérennou DA, Mazibrada G, Chauvineau V, et al. Lateropulsion, pushing and verticality perception in hemisphere stroke: a causal relationship? Brain 2008;131:2401-13.

11 Dai S, Piscicelli C, Clarac E, et al. Balance, lateropulsion, and gait disorders in subacute stroke. Neurology 2021;96:e2147-59.

12 Dai S, Piscicelli C, Clarac E, et al. Lateropulsion after hemispheric stroke: a form of spatial neglect involving Graviception. Neurology 2021;96:e2160-71.

13 Tyson SF, Hanley M, Chillala J, et al. Balance disability after stroke. Phys Ther 2006;86:30-8.

14 Pérennou D, Bénaïm C, Rouget E, et al. [Postural balance following stroke: towards a disadvantage of the right brain-damaged hemisphere]. Rev Neurol 1999;155:281-90.

15 Schmid AA, Van Puymbroeck M, Altenburger PA, et al. Balance and balance self-efficacy are associated with activity and participation after stroke: a cross-sectional study in people with chronic stroke. Arch Phys Med Rehabil 2012;93:1101-7.

16 van der Kooi E, Schiemanck SK, Nollet F, et al. Falls are associated with lower self-reported functional status in patients after stroke. Arch Phys Med Rehabil 2017;98:2393-8.

17 Wesselhoff S, Hanke TA, Evans CC. Community mobility after stroke: a systematic review. Top Stroke Rehabil 2018;25:224-38.

18 Kwong PWH, Ng SSM, Chung RCK, et al. A structural equation model of the relationship between muscle strength, balance performance, walking endurance and community integration in stroke survivors. PLoS One 2017;12:e0185807.

19 Forster A, Young J. Incidence and consequences of falls due to stroke: a systematic inquiry. BMJ 1995;311:83-6.

$20 \mathrm{Xu} \mathrm{T}$, Clemson L, O'Loughlin K, et al. Risk factors for falls in community stroke survivors: a systematic review and meta-analysis. Arch Phys Med Rehabil 2018;99:563-73. 
21 Maeda N, Urabe Y, Murakami M, et al. Discriminant analysis for predictor of falls in stroke patients by using the Berg balance scale. Singapore Med J 2015;56:280-3.

22 Fulk GD, Reynolds C, Mondal S, et al. Predicting home and community walking activity in people with stroke. Arch Phys Med Rehabil 2010;91:1582-6.

23 van de Port IG, Kwakkel G, Lindeman E. Community ambulation in patients with chronic stroke: how is it related to gait speed? $\mathrm{J}$ Rehabil Med 2008;40:23-7.

24 Durcan S, Flavin E, Horgan F. Factors associated with community ambulation in chronic stroke. Disabil Rehabil 2016;38:245-9.

25 Schmid AA, Van Puymbroeck M, Altenburger PA, et al. Balance is associated with quality of life in chronic stroke. Top Stroke Rehabil 2013;20:340-6.

26 Bonan IV, Guettard E, Leman MC, et al. Subjective visual vertical perception relates to balance in acute stroke. Arch Phys Med Rehabil 2006;87:642-6.

27 Bonan IV, Hubeaux K, Gellez-Leman MC, et al. Influence of subjective visual vertical misperception on balance recovery after stroke. J Neurol Neurosurg Psychiatry 2007;78:49-55.

28 Yelnik AP, Lebreton FO, Bonan IV, et al. Perception of verticality after recent cerebral hemispheric stroke. Stroke 2002;33:2247-53.

29 Molina F, Lomas-Vega R, Obrero-Gaitán E, et al. Misperception of the subjective visual vertical in neurological patients with or without stroke: a meta-analysis. NeuroRehabilitation 2019;44:379-88.

30 Barra J, Chauvineau V, Ohlmann T, et al. Perception of longitudinal body axis in patients with stroke: a pilot study. J Neurol Neurosurg Psychiatry 2007;78:43-8.

31 Rossetti Y, Rode G, Pisella L, et al. Prism adaptation to a rightward optical deviation rehabilitates left hemispatial neglect. Nature 1998;395:166-9.

32 Rousseaux M, Honoré J, Vuilleumier P, et al. Neuroanatomy of space, body, and posture perception in patients with right hemisphere stroke. Neurology 2013;81:1291-7.

33 Richard C, Honoré J, Bernati T, et al. Straight-ahead pointing correlates with long-line bisection in neglect patients. Cortex 2004;40:75-83.

34 Chokron S, Bartolomeo P. Correlation between the position of the egocentric reference and right neglect signs in left-brain-damaged patients. Brain Cogn 2000;43:99-104

35 Bonan IV, Leman MC, Legargasson JF, et al. Evolution of subjective visual vertical perturbation after stroke. Neurorehabil Neural Repair 2006;20:484-91.

36 Stone SP, Halligan PW, Greenwood RJ. The incidence of neglect phenomena and related disorders in patients with an acute right or left hemisphere stroke. Age Ageing 1993;22:46-52.

37 Pérennou DA, Leblond C, Amblard B, et al. The polymodal sensory cortex is crucial for controlling lateral postural stability: evidence from stroke patients. Brain Res Bull 2000;53:359-65.

38 Barra J, Oujamaa L, Chauvineau V, et al. Asymmetric standing posture after stroke is related to a biased egocentric coordinate system. Neurology 2009;72:1582-7.

39 Roelofs JMB, van Heugten K, de Kam D, et al. Relationships between Affected-Leg motor impairment, postural asymmetry, and impaired body sway control after unilateral supratentorial stroke. Neurorehabil Neural Repair 2018;32:953-60.

40 Roerdink M, Geurts ACH, de Haart M, et al. On the relative contribution of the paretic leg to the control of posture after stroke. Neurorehabil Neural Repair 2009;23:267-74.

41 van Asseldonk EHF, Buurke JH, Bloem BR, et al. Disentangling the contribution of the paretic and non-paretic ankle to balance control in stroke patients. Exp Neurol 2006;201:441-51.

42 Hugues A, Di Marco J, Ribault S, et al. Limited evidence of physical therapy on balance after stroke: a systematic review and metaanalysis. PLoS One 2019;14:e0221700.

43 Kitago T, Krakauer JW. Motor learning principles for neurorehabilitation. Handb Clin Neurol 2013:110:93-103.

44 Maier M, Ballester BR, Verschure PFMJ. Principles of neurorehabilitation after stroke based on motor learning and brain plasticity mechanisms. Front Syst Neurosci 2019;13:74.

45 Jacquin-Courtois S, O'Shea J, Luauté J, et al. Rehabilitation of spatial neglect by prism adaptation: a peculiar expansion of sensorimotor after-effects to spatial cognition. Neurosci Biobehav Rev 2013;37:594-609.

46 Rode G, Lacour S, Jacquin-Courtois S, et al. Long-term sensorimotor and therapeutical effects of a mild regime of prism adaptation in spatial neglect. A double-blind RCT essay. Ann Phys Rehabil Med 2015;58:40-53.

47 Luauté J, Villeneuve L, Roux A, et al. Adding methylphenidate to prism-adaptation improves outcome in neglect patients. A randomized clinical trial. Cortex 2018:106:288-98.
48 Redding GM, Rossetti Y, Wallace B. Applications of prism adaptation: a tutorial in theory and method. Neurosci Biobehav Rev 2005;29:431-44

49 Prablanc C, Panico F, Fleury L, et al. Adapting terminology: clarifying prism adaptation vocabulary, concepts, and methods. Neurosci Res 2020;153:8-21.

50 O'Shea J, Gaveau V, Kandel M, et al. Kinematic markers dissociate error correction from sensorimotor realignment during prism adaptation. Neuropsychologia 2014;55:15-24.

51 Jeannerod M, Rossetti Y. Visuomotor coordination as a dissociable visual function: experimental and clinical evidence. Baillieres Clin Neurol 1993:2:439-60.

52 Panico F, Rossetti Y, Trojano L. On the mechanisms underlying prism adaptation: a review of neuro-imaging and neuro-stimulation studies. Cortex 2020;123:57-71.

53 Rossetti Y, Jacquin-Courtois S, Calabria M. Testing cognition and rehabilitation in unilateral neglect with wedge prism adaptation: multiple interplays between sensorimotor adaptation and spatial cognition. In: Kansaku K, Cohen LG, Birbaumer N, eds. Clinical systems neuroscience. Tokyo: Springer Japan, 2015: 359-81.

54 Rode G, Rossetti Y, Boisson D. Prism adaptation improves representational neglect. Neuropsychologia 2001;39:1250-4.

55 Girardi M, Mclntosh RD, Michel C, et al. Sensorimotor effects on central space representation: prism adaptation influences haptic and visual representations in normal subjects. Neuropsychologia 2004:42:1477-87.

56 Rode G, Pisella L, Rossetti Y. Bottom-up transfer of sensory-motor plasticity to recovery of spatial cognition: visuomotor adaptation and spatial neglect. In: Progress in brain research. Elsevier, 2003: 273-87.

57 Luauté J, Michel C, Rode G, et al. Functional anatomy of the therapeutic effects of prism adaptation on left neglect. Neurology 2006:66:1859-67.

58 Luauté J, Schwartz S, Rossetti Y, et al. Dynamic changes in brain activity during prism adaptation. $J$ Neurosci 2009;29:169-78.

59 Saj A, Cojan Y, Vocat R, et al. Prism adaptation enhances activity of intact fronto-parietal areas in both hemispheres in neglect patients. Cortex 2013;49:107-19.

60 Lunven M, Rode G, Bourlon C, et al. Anatomical predictors of successful prism adaptation in chronic visual neglect. Cortex 2019;120:629-41.

61 Tilikete C, Rode G, Rossetti Y, et al. Prism adaptation to rightward optical deviation improves postural imbalance in left-hemiparetic patients. Curr Biol 2001;11:524-8.

62 Hugues A, Di Marco J, Lunven M, et al. Long-lasting reduction in postural asymmetry by prism adaptation after right brain lesion without neglect. Cogn Process 2015;16 Suppl 1:371-5.

63 Nijboer TCW, Olthoff L, Van der Stigchel S, et al. Prism adaptation improves postural imbalance in neglect patients. Neuroreport 2014;25:307-11.

64 Shiraishi H, Yamakawa Y, Itou A, et al. Long-term effects of prism adaptation on chronic neglect after stroke. NeuroRehabilitation 2008;23:137-51

65 Padula WV, Nelson CA, Padula WV, et al. Modifying postura adaptation following a CVA through prismatic shift of visuo-spatial egocenter. Brain Inj 2009;23:566-76.

66 Chan A-W, Tetzlaff JM, Altman DG, et al Spirit 2013 statement: defining standard protocol items for clinical trials. Ann Intern Med 2013;158:200.

67 Guillebastre B, Rougier PR, Sibille B, et al. When might a cane be necessary for walking following a stroke? Neurorehabil Neural Repair 2012;26:173-7.

68 Azouvi P, Samuel C, Louis-Dreyfus A, et al. Sensitivity of clinical and behavioural tests of spatial neglect after right hemisphere stroke. $J$ Neurol Neurosurg Psychiatry 2002;73:160-6.

69 Blum L, Korner-Bitensky N. Usefulness of the berg balance scale in stroke rehabilitation: a systematic review. Phys Ther 2008;88:559-66.

70 Ruhe A, Fejer R, Walker B. The test-retest reliability of centre of pressure measures in bipedal static task conditions--a systematic review of the literature. Gait Posture 2010;32:436-45.

71 Sawacha Z, Carraro E, Contessa P, et al. Relationship between clinical and instrumental balance assessments in chronic post-stroke hemiparesis subjects. J Neuroeng Rehabil 2013;10:95

72 Karnath HO, Ferber S, Dichgans J. The origin of contraversive pushing: evidence for a second graviceptive system in humans. Neurology 2000;55:1298-304.

73 Quinn TJ, Langhorne P, Stott DJ. Barthel index for stroke trials: development, properties, and application. Stroke 2011:42:1146-51.

74 Klein A, Andersson J, Ardekani BA, et al. Evaluation of 14 nonlinear deformation algorithms applied to human brain MRI registration. Neuroimage 2009;46:786-802. 
75 Avants BB, Tustison NJ, Song G, et al. A reproducible evaluation of ants similarity metric performance in brain image registration. Neuroimage 2011;54:2033-44.

76 Foulon C, Cerliani L, Kinkingnéhun S, et al. Advanced lesion symptom mapping analyses and implementation as BCBtoolkit. Gigascience 2018;7:1-17.

77 Rorden C, Karnath H-O, Bonilha L. Improving lesion-symptom mapping. J Cogn Neurosci 2007;19:1081-8.

78 Rojkova K, Volle E, Urbanski M, et al. Atlasing the frontal lobe connections and their variability due to age and education: a spherical deconvolution tractography study. Brain Struct Funct 2016;221:1751-66.

79 Thiebaut de Schotten M, Tomaiuolo F, Aiello M, et al. Damage to white matter pathways in subacute and chronic spatial neglect: a group study and 2 single-case studies with complete virtual "in vivo" tractography dissection. Cereb Cortex 2014;24:691-706.

80 Zhang S, Paul J, Nantha-Aree M, et al. Empirical comparison of four baseline covariate adjustment methods in analysis of continuous outcomes in randomized controlled trials. Clin Epidemiol 2014;6:227-35.

81 Pocock SJ, Assmann SE, Enos LE, et al. Subgroup analysis, covariate adjustment and baseline comparisons in clinical trial reporting: current practice and problems. Stat Med 2002;21:2917-30.
82 Twisk J, Bosman L, Hoekstra T, et al. Different ways to estimate treatment effects in randomised controlled trials. Contemp Clin Trials Commun 2018;10:10.1016/j.conctc.2018.03.008:80-5.

83 Liston RAL, Brouwer BJ. Reliability and validity of measures obtained from stroke patients using the balance master. Arch Phys Med Rehabil 1996;77:6-430.

84 Hiengkaew V, Jitaree K, Chaiyawat P. Minimal detectable changes of the berg balance scale, fugl-meyer assessment scale, timed "up \& go" test, gait speeds, and 2-minute walk test in individuals with chronic stroke with different degrees of ankle plantarflexor tone. Arch Phys Med Rehabil 2012;93:1201-8.

85 Flansbjer U-B, Blom J, Brogårdh C. The reproducibility of Berg balance scale and the Single-leg stance in chronic stroke and the relationship between the two tests. $P m R$ 2012;4:10.1016/j. pmrj.2011.11.004:165-70.

86 Alghadir AH, Al-Eisa ES, Anwer S, et al. Reliability, validity, and responsiveness of three scales for measuring balance in patients with chronic stroke. BMC Neurol 2018;18:10.1186/s12883-0181146-9.

87 Higgins J, Thomas J, Chandler J, eds. Cochrane handbook for systematic reviews of interventions version 6.1 (updated September 2020). Cochrane, 2020. www.training.cochrane.org/ handbook 